\title{
Redetermination of the crystal structure of ethylenediammonium chloride, $\mathrm{C}_{2} \mathrm{H}_{10} \mathrm{Cl}_{2} \mathrm{~N}_{2}$
}

\author{
H. Reuter and G. Kastner \\ Universität Osnabrück, Institut für Chemie, Barbarastr. 7, D-49069 Osnabrück, Germany
}

Received August 9, 1996, CSD-No. 402590

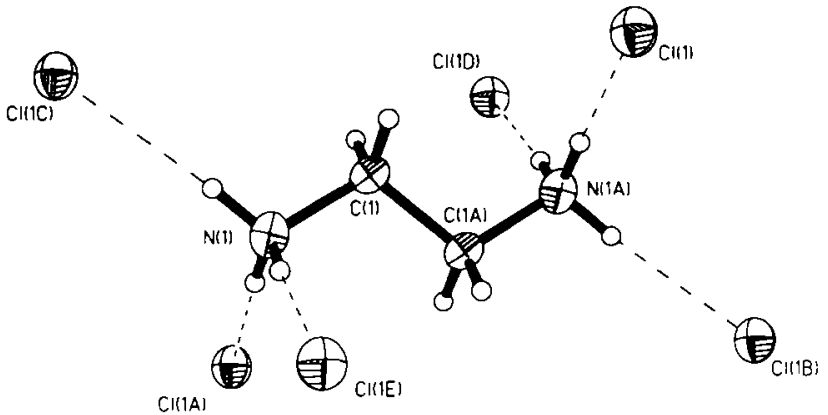

Source of material: The title compound was obtained by reaction of ethylenediamine with $\mathrm{HCl}$ in $\mathrm{DMF} / \mathrm{H}_{2} \mathrm{O}(5: 1)$ solvent.

The crystal structure of ethyeneiammonium chloride first described by T. Ashida and S.Hirokawa (see ref. 1) was redetermined by single crystal $\mathrm{X}$-ray diffraction. The asymmetric unit contains 0.5 formula units. There are two formula units in the monoclinic unit cell. All three hydrogen atoms of the ammonium groups form hydrogen bonds (in the figure indicated by broken lines) to chloride ions. The $\mathrm{N}-\mathrm{Cl}$ distance is $3.183 \AA$, the $\mathrm{H}-\mathrm{Cl}$ distances are $2.296 \AA, 2.267 \AA$ and $2.321 \AA$.

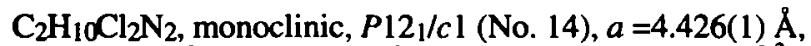
$b=6.888(1) \AA, c=9.958(1) \AA, \beta=91.32(2)^{\circ}, V=303.5 \AA^{3}, Z=2$, $R(F)=0.024, R_{\mathrm{w}}(F)=0.073$.

Table 3. Final atomic coordinates and displacement parameters (in $\AA^{2}$ )

\begin{tabular}{lllllllllll}
\hline Alom & Site & $x$ & $y$ & $z$ & $U_{11}$ & $U_{22}$ & $U_{33}$ & $U_{12}$ & $U_{13}$ & $U_{23}$ \\
\hline $\mathrm{Cl}(1)$ & $4 e$ & $0.09266(6)$ & $0.08003(4)$ & $0.82959(2)$ & $0.0348(2)$ & $0.0295(2)$ & $0.0267(2)$ & $0.00051(8)$ & $-0.0010(1)$ & $0.00150(8)$ \\
$\mathrm{C}(1)$ & $4 e$ & $0.3877(2)$ & $-0.4244(1)$ & $0.9755(1)$ & $0.0221(4)$ & $0.0252(4)$ & $0.0292(5)$ & $0.0034(3)$ & $-0.0013(3)$ & $0.0015(3)$ \\
$\mathrm{N}(1)$ & $4 e$ & $0.5489(2)$ & $-0.2570(1)$ & $0.91642(9)$ & $0.0335(4)$ & $0.0237(4)$ & $0.0285(4)$ & $0.0029(3)$ & $0.0000(3)$ & $0.0013(3)$ \\
\hline
\end{tabular}

Acknowledgments. This work was supported by the Deutsche Forschungsgemeinschaft and the Fonds der Chemischen Industrie.
Table 1. Parameters used for the X-ray data collection

\begin{tabular}{|c|c|}
\hline $\begin{array}{l}\text { Crystal: } \\
\text { Wavelength: }\end{array}$ & $\begin{array}{l}\text { colorless needle, size } 0.15 \times 0.15 \times 0.35 \mathrm{~mm} \\
\text { Mo } K_{\alpha} \text { radiation }(0.71073 \AA)\end{array}$ \\
\hline$\mu:$ & $9.39 \mathrm{~cm}^{-1}$ \\
\hline Diffractometer: & Siemens P4 \\
\hline Scan mode: & $\omega$ \\
\hline $\mathrm{T}_{\text {measurement: }}$ & $293 \mathrm{~K}$ \\
\hline $2 \theta_{\text {max }}:$ & $64^{\circ}$ \\
\hline $\mathrm{N}(\boldsymbol{h} k)_{\text {unique: }}$ & 1058 \\
\hline Criterion for $I_{0}$ : & $I_{0}>2 \sigma\left(I_{0}\right)$ \\
\hline $\mathrm{N}(\text { param })_{\text {refined: }}$ & 34 \\
\hline Program: & SHELXTL \\
\hline
\end{tabular}

Table 2. Final atomic coordinates and displacement parameters (in $\AA^{2}$ )

\begin{tabular}{llllll}
\hline Atom & Site & $x$ & $y$ & $z$ & $U_{\text {iso }}$ \\
\hline H(1A) & $4 e$ & $0.2520(2)$ & $-0.4813(1)$ & $0.9085(1)$ & $0.033(4)$ \\
H(1B) & $4 e$ & $0.2678(2)$ & $-0.3804(1)$ & $1.0500(1)$ & $0.037(4)$ \\
H(1C) & $4 e$ & $0.4150(2)$ & $-0.1686(1)$ & $0.88831(9)$ & $0.054(5)$ \\
H(ID) & $4 e$ & $0.6561(2)$ & $-0.2974(1)$ & $0.84726(9)$ & $0.049(5)$ \\
H(IE) & $4 e$ & $0.6721(2)$ & $-0.2046(1)$ & $0.97829(9)$ & $0.055(5)$ \\
& & & & & \\
\hline
\end{tabular}

\section{References}

1. Ashida, T.; Hirokawa, H.: The Crystal Structure of Ethylenediammonium Chloride. Bull. Chem. Soc. Jpn. 36 (1963) 704-707.

2. Sheldrick, G. M.: Program Package SHELXTL. Release 5. Siemens Analytical X-Ray Instruments Inc., Madison (WI 53719), USA 1994. 